\title{
PrDB: Managing Large-Scale Correlated Probabilistic Databases (Abstract)
}

\author{
Amol Deshpande \\ University of Maryland, College Park, MD, USA \\ amol@cs.umd.edu
}

Increasing numbers of real-world application domains are generating data that is inherently noisy, incomplete, and probabilistic in nature. Statistical inference and probabilistic modeling often introduce another layer of uncertainty on top of that. Examples of such data include measurement data collected by sensor networks, observation data in the context of social networks, scientific and biomedical data, and data collected by various online cyber-sources. Over the last few years, numerous approaches have been proposed, and several systems built, to integrate uncertainty into databases. However, these approaches typically make simplistic and restrictive assumptions concerning the types of uncertainties that can be represented. Most importantly, they often make highly restrictive independence assumptions, and cannot easily model rich correlations among the tuples or attribute values. Furthermore, they typically lack support for specifying uncertainties at different levels of abstractions, needed to handle large-scale uncertain datasets.

In this talk, I will begin by presenting our work on building a probabilistic data management system, called PrDB, aimed at supporting rich correlation structures often present in real-world uncertain datasets. I will present the PrDB representation model, which is based on probabilistic graphical models, and its key abstractions, and show how these enable PrDB to support uncertainties specified at various abstraction levels, from schema-level uncertainties that apply to entire relations to tuple-specific uncertainties that apply only to a specific tuple or a specific set of tuples. Query evaluation in $\mathrm{PrDB}$ can be seen as equivalent to inference in graphical models, and I will present some of the key novel techniques that we have developed to efficiently evaluate various types of queries over largescale probabilistic databases. I will then briefly discuss our ongoing work and some of the open research challenges in this area.

More details about the PrDB system and its key technologies can be found in $[1|2| 3]$.

\section{References}

1. Deshpande, A., Getoor, L., Sen, P.: Graphical Models for Uncertain Data. In: Aggarwal, C. (ed.) Managing and Mining Uncertain Data. Springer, Heidelberg (2008)

2. Kanagal, B., Deshpande, A.: Indexing Correlated Probabilistic Databases. In: SIGMOD (2009)

3. Sen, P., Deshpande, A., Getoor, L.: PrDB: Managing and Exploiting Rich Correlations in Probabilistic Databases. The International Journal on Very Large Data Bases (2009) 